\title{
CARACTERIZAÇÃO ESPECTRAL DE ÁREAS DE GRAMÍNEAS FORRAGEIRAS INFECTADAS COM A DOENÇA "MELA-DAS-SEMENTES DA BRAQUIÁRIA" POR MEIO DE IMAGENS CCD/CBERS-2 ${ }^{1}$
}

\author{
JOSÉ C. ROSATTI², ERIVALDO A. DA SILVA ${ }^{3}$, HÉLIO R. SILVA ${ }^{4}$, \\ PEDRO C. KRUPPA ${ }^{5}$
}

\begin{abstract}
RESUMO: Imagens CCD/CBERS-2, nas bandas espectrais CCD2, CCD3 e CCD4, dos anos de 2004 e 2005, de Mirante do Paranapanema - SP, foram transformadas em reflectância de superfície usando o modelo $5 \mathrm{~S}$ de correção atmosférica e normalizadas radiometricamente. $\mathrm{O}$ objetivo principal foi caracterizar espectralmente áreas de pastagens de Brachiaria brizantha em fase de florescimento, isentas e infectadas com a doença "mela-das-sementes da braquiária", possibilitando a sua detecção por meio da comparação entre os valores de reflectância de superfície denominada de Fator de Reflectância Bidirecional de Superfície (FRBS). Teve-se, também, o objetivo de avaliar a eficácia das imagens CCD/CBERS-2 para a obtenção de respostas espectrais de pastagens. Os dosséis sadios e doentes da Brachiaria brizantha foram identificados por meio da análise dos valores de reflectância e dos dados observados no Índice de Estresse Hídrico Acumulativo Relativo da Cultura (ACWSI) obtidos na área de estudo. Os resultados indicaram que as principais diferenças foram a diminuição da reflectância na banda CCD3 e o aumento da reflectância na banda CCD4 nas áreas doentes. A metodologia empregada com o uso de dados do sensor CCD/CBERS-2, associados ao ACWSI, mostrou-se eficaz para discriminar dosséis infectados com a "mela-das-sementes da braquiária".
\end{abstract}

PALAVRAS-CHAVE: sensoriamento remoto, Brachiaria brizantha, ACWSI.

\section{SPECTRAL CHARACTERIZATION OF FORAGE GRASSES INFECTED WITH THE DISEASE “MELA-DAS-SEMENTES DA BRAQUIÁRIA” THROUGH CCD/CBERS -2 IMAGES}

\begin{abstract}
CCD/CBERS-2 images in the spectral bands of CCD2, CCD3 and CCD4 of the years 2004 and 2005, from Mirante do Paranapanema - SP (Brazil), were transformed into surface reflectance images using the $5 \mathrm{~S}$ atmospheric correction model and radiometrically normalized. The main objective was to spectrally characterize pastures of Brachiaria brizantha in the flowering phase, exempt and infected with the disease "mela-das-sementes da braquiária" making it possible its detection through the comparison among the SBRF - Surface Bidirectional Reflectance Factor values. At the same time, it was aimed to evaluate the effectiveness of the CCD/CBERS-2 images to obtain spectral responses of pastures. The healthy and diseased vegetation canopies were identified through the analysis of the surface reflectance values and the observed data of the Accumulative Crop Water Stress Index (ACWSI) obtained from the study area. The results indicated that the main differences were the decrease of the reflectance in the CCD3 band and the increase of the reflectance in the CCD4 band in the diseased areas. Using the CCD/CBERS-2 data associated with the ACWSI, the methodology was effective to discriminate the vegetation canopies infected with the "mela-das-sementes da braquiária".
\end{abstract}

KEYWORDS: remote sensing, Brachiaria brizantha, ACWSI.

\footnotetext{
${ }^{1}$ Extraído da dissertação de mestrado do primeiro autor.

${ }^{2}$ Eng $^{\mathrm{o}}$ Agrônomo, Programa de Pós-Graduação em Ciências Cartográficas, UNESP, R. Roberto Simonsen, 305, Presidente Prudente - SP, jcrost@gmail.com

${ }^{3}$ Eng $^{\mathrm{e}}$ Cartógrafo, Prof. Dr., Programa de Pós-Graduação em Ciências Cartográficas, UNESP, Presidente Prudente - SP, erivaldo@prudente.unesp.br

${ }^{4}$ Eng ${ }^{\mathrm{o}}$ Agrônomo, Prof. Dr., Departamento de Fitossanidade, Engenharia Rural e Solos, UNESP, Ilha Solteira - SP, hrsilva@agr.feis.unesp.br

${ }^{5}$ Engo Agrônomo, Pesquisador, Instituto Biológico/CPDSV, São Paulo - SP, pckruppa@ biologico.sp.gov.br

Recebido pelo Conselho Editorial em: 2-5-2006
}

Aprovado pelo Conselho Editorial em: 6-9-2006 


\section{INTRODUÇÃO}

A base da alimentação animal nos trópicos são as pastagens e, no Brasil, representam área estimada de 197 milhões de hectares (FAO, 2005), compondo dois importantes segmentos do agronegócio brasileiro: a produção de carne e a produção de sementes de forrageiras que, juntas, ocupam boa parcela das exportações de produtos agropecuários do País.

Devido à boa adaptabilidade sob diferentes condições climáticas, à tolerância aos solos de baixa fertilidade e ácidos, e também à resistência ao manejo inadequado dos sistemas de produção extensivos no Brasil, nessas duas últimas décadas, tem sido considerável o aumento das áreas de pastagens com o gênero Brachiaria. Todavia, a baixa variabilidade genética das principais espécies cultivadas (B. brizantha e B. decumbens) e o seu modo de reprodução apomítico (assexuado) resultou em alta homogeneidade na população de gramíneas nas pastagens implantadas, constituindo, dessa forma, extensos monocultivos, facilitando o aparecimento e a propagação de pragas e doenças, ocasionando perdas consideráveis à produção de sementes e de forragens.

Entre as doenças mais importantes, destaca-se a "mela-das-sementes da braquiária", causada pelo fungo Claviceps sulcata Langdon (forma teleomórfica de Sphacelia sp.), que afeta os ovários das flores de plantas de B. brizantha (Hoschst ex A. Rich) Stapt, causando a infertilidade do pólen. Sua epidemiologia é ainda desconhecida, acreditando-se que a disseminação do patógeno seja efetuada principalmente por insetos e por gotas de chuva (VERZIGNASSI \& FERNANDES, 2001) e, por isso, favorecida em épocas mais chuvosas ou nubladas.

A incidência da doença dá-se na fase reprodutiva das gramíneas, que vai do florescimento até a colheita das sementes. As características dessa doença podem ser descritas a partir de sua fase inicial, quando ela é reconhecida pela presença de gotas de líquido pegajoso, contendo conídios do fungo, de coloração inicialmente rosada e, mais tarde, parda (REIS et al., 2000), que exsudam dos ovários infectados e, ao se depositarem nas folhas das plantas, tornam-nas brancas devido à presença de açúcares. Por se tratar de doença de incidência recente nas áreas de pastagens e em campos de produção de sementes de forrageiras do País, poucos trabalhos de pesquisa foram realizados sobre a mesma, tornando escassas as informações disponibilizadas sobre o seu comportamento e o controle do patógeno.

Por outro lado, o Sensoriamento Remoto é uma ferramenta que possibilita a extração, a distância, de informações de alvos de interesse na superfície da Terra. Porém, a obtenção da resposta espectral do dossel de uma cultura pode ser influenciada por diversos fatores, tais como: a geometria de iluminação e a visada (parâmetros geométricos que caracterizam a bidirecionalidade da radiância medida pelos sensores remotos), a umidade, o vigor vegetativo, a cobertura vegetal do substrato, o tipo de solo, o relevo, o espaçamento da cultura, a variedade, a idade da planta, entre outros (PONZONI et al., 2002). GOMIDE \& ASSAD (1990) recomendam, para a identificação de pastagem, a utilização de imagens orbitais obtidas na mesma fenofase do ciclo dessa cultura. MUTANGA et al. (2004) utilizaram o espectrorradiômetro para identificar as feições de absorção de macronutrientes e assim prognosticar o nível nutricional de pastagens naturais. Os autores recomendam para a identificação da qualidade nutricional das pastagens, por meio do sensoriamento remoto orbital, a utilização das bandas espectrais correspondentes ao vermelho e ao infravermelho médio.

Além disso, deve-se ainda considerar que os sinais eletromagnéticos da radiação coletados pelos sensores remotos a bordo de satélites, no espectro solar, são modificados, dispersos e absorvidos por gases e aerossóis por meio de sua passagem pela atmosfera, no caminho entre a superfície da terra e o sensor (SONG et al., 2001).

Dessa forma, o objetivo principal deste trabalho foi estabelecer metodologia que permitisse a caracterização espectral de dosséis de pastagens $B$. brizantha cv MG-5 sadios e infectados pela doença "mela-das-sementes da braquiária", por meio da análise das respostas espectrais desses alvos, obtidas dos valores de Fatores de Reflectância Bidirecional de Superfície (FRBS) a partir das imagens multitemporais associadas à análise do Índice de Estresse Hídrico Acumulativo Relativo 
da Cultura (ACWSI). Teve-se também o objetivo de avaliar o potencial de emprego de imagens CCD/CBERS-2 para o levantamento de áreas de pastagens.

\section{MATERIAL E MÉTODOS}

O estudo foi realizado no município de Mirante do Paranapanema, a $56 \mathrm{~km}$ de Presidente Prudente - SP, na região oeste do Estado de São Paulo, em área situada dentro do quadrilátero limitado pelas latitudes $22^{\circ} 10^{\prime} \mathrm{S}$ e $22^{\circ} 17^{\prime} \mathrm{S}$ e longitudes $51^{\circ} 52^{\prime} \mathrm{W}$ e $51^{\circ} 45^{\prime} \mathrm{W}$ (Figura 1 ). A região é dominada por relevo suavemente ondulado, solos com média e baixa fertilidade, com predominância dos Latossolos (EMBRAPA, 1999), e cobertura vegetal ocupada predominantemente por pastagens implantadas, culturas anuais de algodão, milho, feijão de inverno e com poucos e pequenos fragmentos de matas nativas.

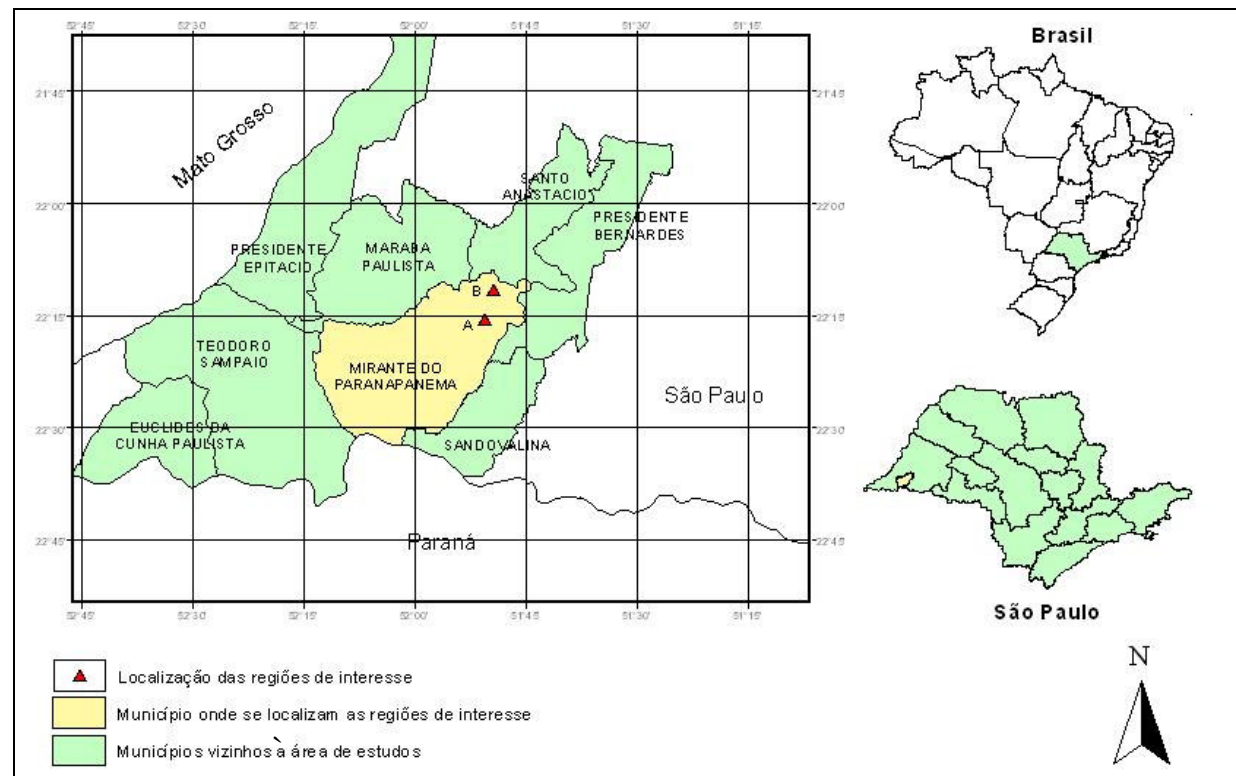

FIGURA 1. Localização da área de estudo.

O clima é tropical de altitude (Cwa), com inverno seco e verão úmido. É uma região de grande concentração de plantio de gramíneas forrageiras destinadas à produção de sementes e, por conseqüência, com histórico de surgimento e danos causados pela doença "mela-das-sementes da braquiária".

Dentro da área de estudo, foram selecionadas duas regiões de interesse, levando em consideração o conhecimento prévio do local e a disposição dos elementos que compõem a cobertura superficial da cena, encontrando-se ambas, no mesmo tipo de solo e relevo plano, evitando-se, assim, a maior parte da interferência da variação topográfica e, por conseguinte, as variações de intensidade de energia detectadas pelos sensores e causadas pelas mudanças dos ângulos de visada.

As áreas de pastagens estudadas foram: a) área de 56 ha, sendo as coordenadas geográficas (ponto central da área): $51^{\circ} 50^{\prime} 46^{\prime \prime W}$ e $22^{\circ} 15^{\prime} 50^{\prime \prime} \mathrm{S}$; e b) área de 17 ha com as coordenadas


Brizantha cv MG-5 e distantes entre si de, aproximadamente, $10 \mathrm{~km}$.

\section{Obtenção de dados orbitais}

Foram obtidas imagens do sensor CCD/CBERS 2 em formato GEOTIFF, nível de processamento 2 e os aplicativos SPRING 4.1.1 e IMPIMA 4.1.1, cedidos pelo INPE, Instituto Nacional de Pesquisas Espaciais (http://www.inpe.br). Na Tabela 1, apresentam-se os dados relevantes referentes às imagens que cobriram a área de estudo. 
TABELA 1. Dados das imagens orbitais empregadas no estudo.

\begin{tabular}{lcc}
\hline \multicolumn{1}{c}{ Dados } & CBERS-2/CCD (1) & CBERS-2/CCD (2) \\
\hline Órbita/ponto & $160 / 125$ & $160 / 125$ \\
Data da passagem do satélite sobre a área de estudo & $13-6-2004$ & $17-5-2005$ \\
Bandas espectrais & CCD2, CCD3 e CCD4 & CCD2, CCD3 e CC4 \\
Elevação solar & $37,2346^{\circ}$ & $40,8178^{\circ}$ \\
Azimute solar & $31,9683^{\circ}$ & $34,1132^{\circ}$ \\
Resolução espacial (m) & 20 & 20 \\
\hline
\end{tabular}

Fonte: INPE - Instituto Nacional de Pesquisas Espaciais (2005).

\section{Caracterização espectral das pastagens}

Os parâmetros biofísicos relativos à cultura estudada foram obtidos na fenofase denominada de florescimento, época de ocorrência da doença "mela-das-sementes da braquiária". As variáveis analisadas foram percentagem de cobertura vegetal (COV) e altura das plantas, além do efeito do estresse hídrico. Essas variáveis foram analisadas com o objetivo de verificar as suas interferências nas respostas espectrais dos alvos ou o grau de intensidade de suas influências sobre os valores de reflectância de superfície das áreas A e B.

A ocorrência do estresse hídrico foi verificada por meio do Índice de Estresse Hídrico Acumulativo Relativo da Cultura (ACWSI). Esse índice é calculado por meio da utilização dos valores referentes à evapotranspiração real, à evapotranspiração potencial e à água disponível no solo (CIIAGRO, 2005), dados obtidos a partir do Balanço Hídrico de Mirante do Paranapanema de 2004 e 2005.

\section{Processamento das imagens CCD/CBERS-2}

No processamento digital das imagens, foram utilizados os processos de correção atmosférica e de normalização relativa, para ajustarem a geometria e a radiometria dos alvos semelhantes, a fim de possibilitar a comparação entre eles, tendo em vista que as imagens foram obtidas em datas diferentes e apresentam-se sob diferentes condições atmosféricas e de iluminação.

Após a aquisição das imagens, foi gerado um banco de dados de imagens em meio digital para as bandas CCD2, CCD3 e CCD4. Sobre as imagens inseridas no banco de dados, foram aplicados os procedimentos de pré-processamento, ou seja, recorte da área de estudo e registro. $\mathrm{Na}$ seqüência, foram aplicados sobre as mesmas os processos de correção atmosférica, de conversão dos NDs das imagens para Fatores de Reflectância Bidirecional Aparente (FRBA) e de normalização radiométrica. A normalização radiométrica foi feita para amenizar as distorções radiométricas das imagens temporais, inclusive as causadas pelas atenuações atmosféricas, garantindo a compatibilidade radiométrica entre as imagens. Para tanto, foi utilizada a metodologia desenvolvida por HALL et al. (1991), que se fundamenta na constituição de regressões lineares estabelecidas mediante a identificação de uma série de controles radiométricos constituídos por valores de FRBS oriundos de objetos pseudoinvariantes claros e escuros. Para a escolha dos objetos pseudoinvariantes, foram originadas duas imagens da transformação "Tasseled Cap", ou seja, "Brighteness e Greenness" (KAUTH \& THOMAS, 1976), que fornecem os dados de entrada do modelo de normalização radiométrica; para tanto, foram utilizados os coeficientes apresentados por CRIST \& CICONE (1984), conforme descrito em FRIEDL et al. (1994). A utilização desses coeficientes na normalização de imagens do sensor CCD justifica-se devido à inexistência de dados publicados para esse sensor.

A imagem "2" (Tabela 1) foi utilizada como "referência" por ter sido obtida em melhores condições de visibilidade, sendo sobre a mesma aplicado o processo de correção atmosférica por meio do modelo 5S (Simulação do Sinal do Satélite dentro do Espectro Solar), descrito em TANRÉ et al. (1992), para o qual foi utilizado o programa SCORADIS - Sistema de Correção Radiométrica de Imagens de Satélites (Tabela 2). Assim, cada banda da imagem original foi transformada em "imagem reflectância de superfície", obtendo-se, com isso, os valores de FRBS. 
TABELA 2. Descrição dos parâmetros usados na correção atmosférica pelo aplicativo SCORADIS.

\begin{tabular}{lcll}
\hline Dia e mês da aquisição da imagem: & $17-5-2005$ & Modelo de aerossol: & Continental \\
Hora decimal universal: & 13,595 & Concentração de aerossóis: & a partir da visibilidade \\
Latitude e Longitude no centro da cena: & $-51,812 /-22,222$ & Visibilidade: & 30 km \\
Modelo atmosférico: & tropical & Seleção de bandas: & CCD2, CCD3 e CCD4 \\
\hline
\end{tabular}

Na imagem "1", de 13-6-2004, denominada "ajuste", os valores de NDs foram inicialmente convertidos para FRBA por meio de rotina escrita em linguagem LEGAL - Linguagem Espacial para Geoprocessamento Algébrico (CÂMARA et al., 1996), própria do aplicativo SPRING 4.1.1 e posteriormente, sobre elas, aplicado o processo de normalização radiométrica.

Posteriormente, procedeu-se à visualização e à avaliação da qualidade da normalização das imagens por meio da comparação entre as médias dos valores de FRBS dos pontos claros e escuros da imagem "referência" e da imagem "ajuste", antes e depois da normalização. Vale dizer que os valores de FRBA da imagem "ajuste" passam a ser considerados como FRBS devido à promoção da compatibilidade radiométrica.

Na sequiência, em cada banda das imagens, foram extraídas as médias dos valores de FRBS dos pixels que formam cada uma das áreas, denominadas A e B. Essa etapa do trabalho foi feita no ambiente SPRING, por meio da geração de categoria temática contendo um plano de informação para cada uma das áreas, em que foram inseridos os polígonos via edição vetorial, a partir dos quais foram obtidos os parâmetros estatísticos utilizados na análise comparativa.

\section{RESULTADOS E DISCUSSÃO}

O processo de obtenção das imagens levou em consideração os períodos de máximo florescimento das plantas e de incidência da doença "mela-das-sementes da braquiária", nas áreas A e B, como pode ser visto na Tabela 3.

TABELA 3. Dados das imagens CCD e das condições fitossanitárias da Brachiaria brizantha nas áreas A e B nas datas de passagem do satélite CBERS-2 sobre a área de estudo.

\begin{tabular}{cccccc}
\hline Imagem & Área & Plantio & Florescimento & Doença & Data da imagem \\
\hline 1 & A, B & out.2003 & jun.2004 & Sim & $13-6-2004$ \\
2 & A & out.2004 & jun.2005 & Não & $17-5-2005$ \\
2 & B & out.2003 & mai.2005 & Não & $17-5-2005$ \\
\hline
\end{tabular}

$\mathrm{Na}$ análise das variáveis biofísicas, verificou-se que, nas datas de passagem do satélite, as pastagens de Brachiaria das áreas A e B encontravam-se com a cobertura vegetal (COV) próxima a $100 \%$, com a altura média das plantas situando-se em torno de $1,63 \mathrm{~m}$, resultando em dosséis homogêneos. Do mesmo modo, a distribuição espacial dos elementos da vegetação e as orientações das plantas nos dosséis foram praticamente iguais para as duas áreas e em ambos os períodos de estudo. Dessa forma, considerando-se que não houve variação significativa na produção de biomassa durante os ciclos das culturas e que a densidade dos elementos da vegetação foi uniforme, ou seja, a cobertura vegetal (COV) e a altura das plantas nas duas áreas estudadas foram semelhantes nos dois períodos agrícolas, assumiu-se que não houve alterações substanciais na reflectância dos dosséis em função desses parâmetros biofísicos.

O balanço hídrico de 2004 do município de Mirante do Paranapanema demonstrou que as condições de suprimento da água no solo nesse ano foram amplamente satisfeitas. Em 2005, o balanço hídrico indicou que, no período de florescimento das pastagens, os valores da disponibilidade hídrica (DH) foram inferiores a 0, 4 (Tabela 4), significando baixa disponibilidade de água para as plantas. 
TABELA 4. Balanço hídrico do município de Mirante do Paranapanema em 2005.

\begin{tabular}{ccrccccccccc}
\hline \multirow{2}{*}{ Período } & $\begin{array}{c}\mathrm{T} \\
\mathrm{C}\end{array}$ & $\begin{array}{c}\mathrm{P} \\
\mathrm{mm}\end{array}$ & $\begin{array}{c}\text { ETP } \\
\mathrm{mm}\end{array}$ & P-ETP & NEG-AC & $\begin{array}{c}\text { ARM } \\
\mathrm{mm}\end{array}$ & $\begin{array}{c}\text { ALT } \\
\mathrm{mm}\end{array}$ & $\begin{array}{c}\text { ETR } \\
\mathrm{mm}\end{array}$ & $\begin{array}{c}\text { DEF } \\
\mathrm{mm}\end{array}$ & $\begin{array}{c}\text { EXC } \\
\mathrm{mm}\end{array}$ & DH \\
\hline 2-5-2005 a 8-5-2005 & 22,1 & 0,0 & 17,8 & $-17,8$ & $-103,3$ & 35,6 & $-7,0$ & 7,0 & 10,9 & 0,0 & 0,39 \\
9-5-2005 a 15-5-2005 & 24,7 & 0,0 & 23,9 & $-23,9$ & $-127,3$ & 28,0 & $-7,6$ & 7,6 & 16,3 & 0,0 & 0,32 \\
$16-5-2005$ a 22-5-2005 & 26,4 & 0,0 & 28,4 & $-28,4$ & $-155,6$ & 21,1 & $-6,9$ & 6,9 & 21,5 & 0,0 & 0,24 \\
23-5-2005 a 29-5-2005 & 22,6 & 63,0 & 18,4 & 44,6 & $-42,1$ & 65,7 & 44,6 & 18,4 & 0,0 & 0,0 & 1,00 \\
\hline
\end{tabular}

Fonte: Elaborado a partir de dados do Centro Integrado de Informações Agrometeorológicas - CIIAGRO (2005).

T - temperatura; P - precipitação; ETP - evapotranspiração potencial; NEG-AC - negativo acumulado; ARM armazenamento da água no solo; ALT - alteração do armazenamento; ETR - evapotranspiração real; DEF - deficiência hídrica; EXC - excedente hídrico; DH - disponibilidade hídrica.

A partir dos dados de DH, foi calculado o valor do índice ACWSI para o período que vai de 15-5-2005 a 18-5-2005, pois a obtenção da imagem " 2 " foi em 17-5-2005. Com isso, foi possível detectar estresse hídrico na área de estudo, nesse período (Tabela 5).

TABELA 5. Condições para desenvolvimento vegetal acumulativo da Brachiaria brizantha.

\begin{tabular}{|c|c|c|c|}
\hline \multicolumn{4}{|c|}{ Condições Médias de Estresse Hídrico da Cultura: 15-5-2005 a 18-5-2005 } \\
\hline Local & Profundidade $(\mathrm{cm})$ & ACWSI & Condições \\
\hline Mirante do Paranapanema & 25 & 1,00 & Críticas \\
\hline Mirante do Paranapanema & 50 & 0,85 & Críticas \\
\hline Mirante do Paranapanema & 75 (pastagens) & 0,85 & Críticas \\
\hline Mirante do Paranapanema & 100 & 0,85 & Críticas \\
\hline
\end{tabular}

Fonte: Adaptado do Centro Integrado de Informações Agrometeorológicas - CIIAGRO (2005).

A correção da interferência atmosférica realizada apenas na imagem de "referência" apresentou resultados satisfatórios nas bandas CCD2, CCD3 e CCD4, como pode ser observado pela análise do gráfico apresentado na Figura 2, referente aos valores de FRBS de um alvo invariante escuro (massa de água).

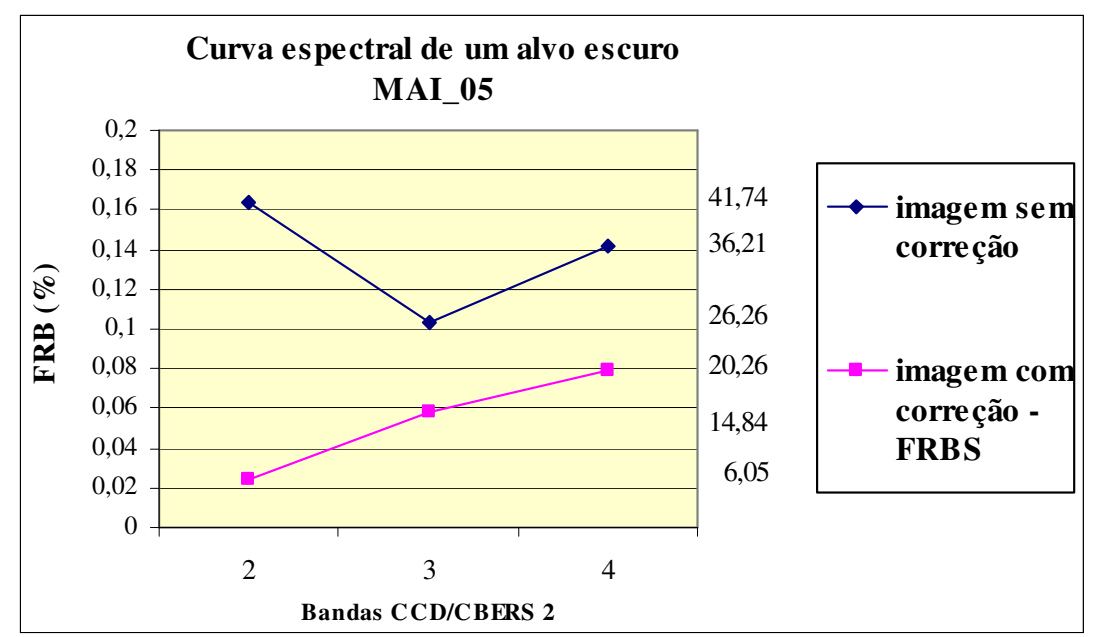

FIGURA 2. Comparação de dados da imagem MAI_05 de 17-5-2005 "referência" antes da correção atmosférica (imagem sem correção) e após a aplicação da correção atmosférica (imagem com correção-FRBS).

Os resultados da aplicação da normalização radiométrica são observados na Figura 3, na qual são comparadas as médias de valores de FRBS dos pontos invariantes claros (solo exposto) da imagem "MAI_05_média_claros" (referência) com as médias da imagem "JUN_04_média_claros" (ajuste) de 13-6-2004, após essa última ter sido normalizada.

Pelo gráfico, podem ser vistas diferenças significativas entre as curvas das respostas espectrais obtidas das médias dos valores de FRBS dos pontos da imagem 
"MAI_05_média_claros" (referência) e da imagem "JUN_04_média_claros" (ajuste). Porém, após o processo de normalização, essa diferença decresceu, ficando os valores bem próximos entre si, como pode ser observado pela curva de resposta espectral resultante da imagem “JUN_04_média_claros_retif", indicando, assim, que o processo realizado foi bem-sucedido.

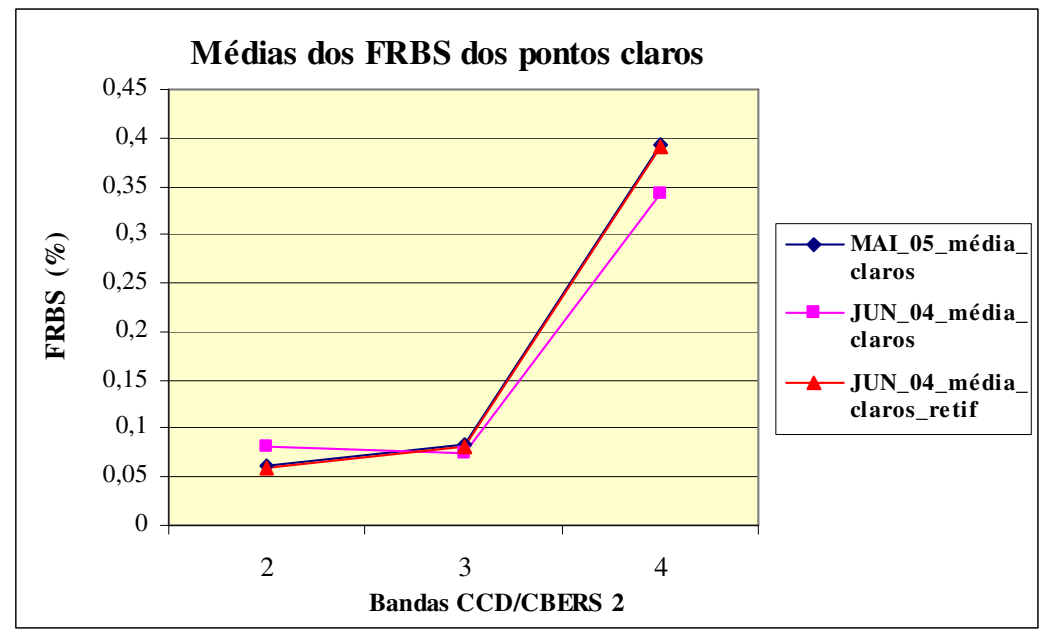

FIGURA 3. Resultado da normalização da imagem "JUN_04_média_claros" de 13-6-2004.

Após a normalização radiométrica, as amostras que correspondem às áreas $\mathrm{A}$ e $\mathrm{B}$ foram delimitadas, resultando, respectivamente, em 320 e 130 pixels. Depois disso, foram extraídos os valores médios de FRBS dos pixels dos dosséis das pastagens de Brachiaria brizantha, conforme consta da Tabela 6. Dessa forma, para cada banda espectral, um único valor de FRBS passou a representar a área da pastagem estudada.

TABELA 6. Valores médios de FRBS coletados nos dois dosséis das pastagens.

\begin{tabular}{cccc}
\hline & \multicolumn{3}{c}{ Valores Médios de FRBS } \\
\cline { 2 - 4 } Bandas & Área_A & Área_B & Área_B \\
CCD & JUN_04 & JUN_04 & MAI_05 \\
& Doente & Doente & Sadia \\
\hline CCD2 & 0,055 & 0,058 & 0,054 \\
CCD3 & 0,061 & 0,069 & 0,076 \\
CCD4 & 0,317 & 0,325 & 0,296 \\
\hline
\end{tabular}

As caracterizações espectrais das pastagens estão definidas na Figura 4, na qual se verifica que as curvas das áreas doentes mostram boa similaridade entre si, porém descrevem diferenças espectrais significativas nas bandas CCD3 e CCD4, quando a comparação é feita entre dosséis doentes das áreas A e B, observados na imagem de 13-6-2004, e o dossel sadio da área B, observado na imagem de 17-5-2005.

$\mathrm{Na}$ banda CCD3, que corresponde à radiação vermelha do espectro visível, observa-se aumento no valor de FRBS da área sadia em relação às áreas doentes, possivelmente devido à ocorrência, em maio de 2005, de estresse hídrico na área B. Isso é corroborado por MOREIRA (2003), que constatou, para a cultura de trigo, aumento da reflectância do dossel nessa região espectral, em função da deficiência hídrica. Portanto, a alteração da reflectância na banda CC3, observada nesses dosséis, minimizou a separabilidade espectral em relação aos dosséis com a doença "mela".

Os resultados também demonstram que a presença da doença nas áreas A e B, em 2004, provavelmente foi responsável pelo aumento dos valores de FRBS no infravermelho próximo (CCD4), em função do efeito causado pela doença à estrutura interna da planta. 


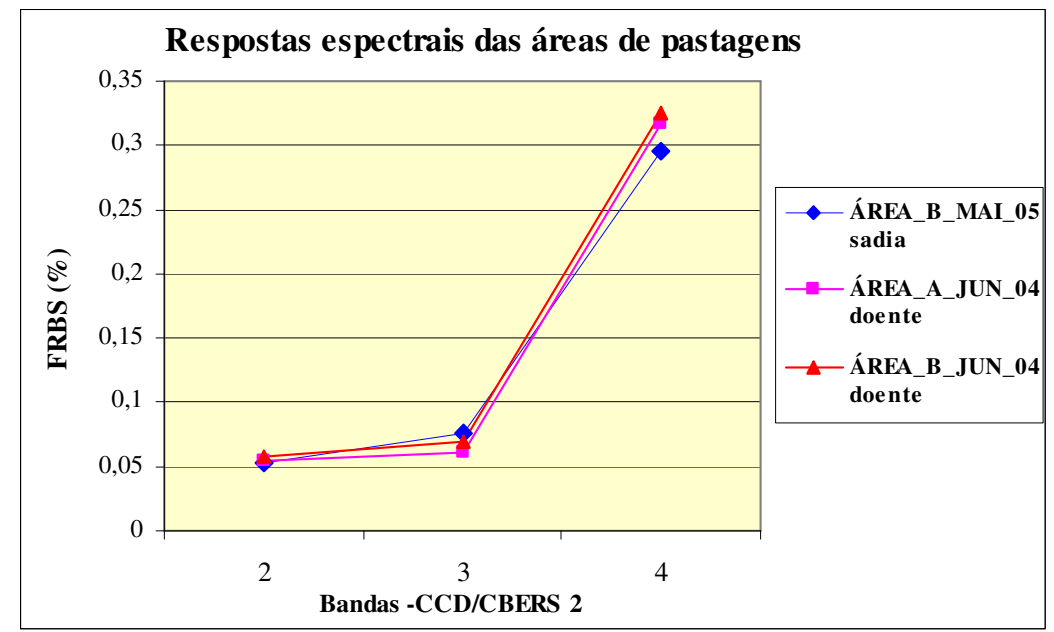

FIGURA 4. Comparação entre valores de FRBS dos dosséis doentes nas áreas A e B observados na imagem de 13-6-2004 (ÁREA_A_JUN_04doente e ÁREA_B_JUN_04doente), e do dossel sadio na área B, observado na imagem de 17-5-2005 (ÁREA_B_MAI_05sadia).

A análise estatística foi inicialmente feita entre as áreas B de 2004 e 2005, consideradas amostras dependentes, com a aplicação do teste de ANDERSON-DARLING, que avaliou a normalidade presente nos valores de FRBS de cada área nas três bandas. Dessa maneira, foi constatado que apenas os valores da banda CCD4 da área B apresentaram distribuição normal. Devido a isso, na comparação entre o dossel com sintomas da "mela-das-sementes da braquiária" da área B, em junho de 2004, e o dossel sadio da pastagem da área B, em maio de 2005, foi utilizado o teste paramétrico $t$ pareado (para pares de observação), que investigou a igualdade entre a banda CCD4 da área B de 2005 com a banda CCD4 da área B de 2004, e o teste não-paramétrico de Wilcoxon (para dados pareados) para testar a igualdade entre as bandas CCD2 de 2004 e CCD2 de 2005 e as bandas CCD3 de 2004 e CCD3 de 2005 da área B.

Pela análise da aplicação da distribuição $t$ de Student (Tabela 7), observa-se que, na comparação entre valores médios de FRBS da banda CCD4 da área B, de junho 2004 e maio 2005, os valores de FRBS foram significativamente diferentes.

TABELA 7. Comparação entre valores médios de FRBS da banda CCD-4 dos dosséis instalados nas áreas B de junho 2004 e maio 2005.

\begin{tabular}{cccc}
\hline \multirow{2}{*}{ Bandas } & \multicolumn{2}{c}{ Valores Médios de FRBS } & \multirow{2}{*}{ Valor de t } \\
\cline { 2 - 3 } CCD & Área_B & Área_B & Student \\
& JUN_04 & MAI_05 & Sadia \\
& Doente & 0,296 & $30,40^{*}$ \\
\hline CCD4 & 0,325 &
\end{tabular}

* Diferença significativa a $0,1 \%$

Os resultados da aplicação do teste de Wilcoxon (Tabela 8), que verificou a existência de diferenças significativas entre as bandas CCD2 de 2004 e CCD2 de 2005 e entre CCD3 de 2004 e CCD3 de 2005 da área B, mostram que ficou rejeitada a hipótese nula de igualdade (p-valor < 0,001), estabelecendo, com isso, que os valores médios de FRBS diferem significativamente a $0,1 \%$ nas bandas analisadas.

Para a comparação entre o dossel doente da área A, de junho de 2004, e o dossel sadio da área B, de maio de 2005, foi aplicado o teste de Mann Whitney (não-paramétrico para amostras independentes). Nesse caso, foram observados os pares das três bandas (CCD2, CCD3 e CCD4) de ambas as imagens (Tabela 9).

De acordo com o resultado do teste, verificou-se que há diferença significativa entre os valores de reflectância nas três bandas das duas imagens ( $\mathrm{p}$-valor $<0,0001$ ). 
TABELA 8. Comparação entre valores médios de FRBS das áreas B de junho 2004 e maio 2005.

\begin{tabular}{cccc}
\hline & \multicolumn{2}{c}{ Valores Médios de FRBS } & \\
\cline { 2 - 3 } Bandas & Área_B & Área_B & p-valor \\
CCD & JUN_04 & MAI_05 & \\
& Doente & Sadia & $<0,001$ \\
CCD2 & 0,058 & 0,054 & $<0,001$ \\
\hline CCD3 & 0,069 & 0,076 & \\
\hline
\end{tabular}

TABELA 9. Comparação entre valores médios de FRBS entre as áreas A de junho 2004 e área B de maio 2005.

\begin{tabular}{cccc}
\hline & \multicolumn{2}{c}{ Valores Médios de FRBS } & \\
\cline { 2 - 3 } Bandas & Área_A & Área_B & p-valor \\
CCD & JUN_04 & MAI_05 & \\
& Doente & Sadia & $<0,0001$ \\
CCD2 & 0,055 & 0,054 & $<0,0001$ \\
CCD3 & 0,061 & 0,076 & $<0,0001$ \\
CCD4 & 0,317 & 0,296 & \\
\hline
\end{tabular}

\section{CONCLUSÕES}

É possível distinguir espectralmente áreas de pastagens de $B$. brizantha cv MG-5 sadias e das áreas com incidência da doença "mela-das-sementes da braquiária", a partir de imagens do sensor CCD associadas ao ACWSI.

Nas comparações feitas entre os valores de FRBS obtidos, as diferenciações mais importantes foram a diminuição da reflectância na banda CCD3 e o aumento da reflectância na banda CCD4 das áreas doentes em relação à área sadia.

A ocorrência de estresse hídrico na pastagem da área B, no ano de 2005, ocasionando alterações na reflectância do dossel, explicou maior valor de FRBS na banda CCD3 da área sadia, e os sintomas da doença "mela-das-sementes da braquiária" provavelmente foram os responsáveis pelos maiores valores de FRBS na banda CCD4, em relação à área sadia.

Embora a associação de dados espectrais ao ACWSI tenha possibilitado a identificação de dosséis sadios e com os sintomas da "mela-das-sementes da braquiária", estudos voltados ao entendimento de tais relações devem ser aprofundados.

\section{REFERÊNCIAS}

CÂMARA, G.; SOUZA, R.C.M.; FREITAS, U.M.; GARRIDO, J. Spring: integrating remote sensing and GIS by object-oriented data modeling. Computer \& Graphics, New York, v.20, n.3, p.395-403, 1996.

CIIAGRO. Centro Integrado de Informações Agrometeorológicas. Disponível em:

< http://www.iac.sp.gov.br/Ciiagro/>. Acesso em: 7 dez. 2005.

CRIST, E.P.; CICONE, R.C. A physically-based transformation of Thematic Mapper data. The TM tasseled cap. IEEE Transactions on Geoscience and Remote Sensing, Picataway, v.GE-22, n.3, p.256-63, 1984.

EMBRAPA. Centro Nacional de Pesquisa de Solos. Sistema brasileiro de classificação de solos. Brasília-1999. 412 p.

FAO. Statistical databases: Faostat. Disponível em: $<$ http://www.fao.org $>$. Acesso em: 10 jun. 2005. 
FRIEDL, M.; MICHAELSON, J.; DAVIS, F.W.; WALKER, H.; SCHIMEL, D.S. Estimating grassland biomass and leaf area index using ground and satellite data. International Journal of Remote Sensing, London, v.15, n.7, p.1401- 20, 1994.

GOMIDE, C.C.C.; ASSAD, E.D. Avaliação da distribuição do Andropon gayanus cv. Planaltina utilizando imagens de satélites TM-Landsat em áreas de cerrado. Pasturas Tropicales, Cali, v.12, n.3, p.2-6, 1990 .

HALL, F.G.; STREBEL, D.E.; NICKESON, J.E.; GOETZ, S.J. Radiometric rectification: toward a common radiometric response among multidate, multisensor images. Remote Sensing of Environment, New York, v.35, n.1, p.11-27, jan. 1991.

INPE. INSTITUTO NACIONAL PESQUISAS ESPACIAIS. Sistema de Processamento de Informações Georreferenciadas (SPRING versão 4.1.1) [on line] Disponível em: <www.dpi.inpe.br/spring >. Acesso em: 10 jun. 2005.

KAUTH, R.J.; THOMAS, G.S. The tasseled cap - a graphic description of the spectral-temporal development of agricultural crops as seen in Landsat. In: SYMPOSIUM ON MACHINE PROCESSING OF REMOTELY SENSED DATA, 1976, West Lafayette, Indiana. Proceedings... p.41-51.

MOREIRA, M.A. Fundamentos do sensoriamento remoto e metodologias de aplicação. 2.ed. Viçosa: UFV, 2003. 307 p.

MUTANGA, O.; SKIDMORE, A.K.; PRINS, H.H.T. Predicting in situ pasture quality in the Kruger National Park, South Africa, using continuum-removed absorption features. Remote Sensing of Environment, New York, v.89, n.3, p.393-408, 2004.

PONZONI, F.J.; GOMES, A.R.; MALDONADO, F.D.; SASSAGAWA, S.H.Y.; NUMATA, I.; ARAÚJO, L.S. Comparação entre o fator de reflectância bidirecional de um dossel de mudas de Eucalyptus grandis e o fator de reflectância direcional hemisférico de suas folhas isoladas. Cerne, Lavras, v.8, n.1, p.59-68, 2002.

REIS, E.M.; CASA, R.T.; BLUM, M.M.C. Doença açucarada do sorgo. Fitopatologia Brasileira, Brasília, v.25, n.3, p.479-90, 2000.

SONG, C.; WOODCOCK, C.E.; SETO, K.C.; LENNEY, M.P.; MACOMBER, S.A. Classification and change detection using Landsat TM data: when and how to correct atmospheric effects? Remote Sensing of Environment, New York, v.75, n.2, p.230-44, 2001.

TANRÉ, D.; HOLBEN, B.N.; KAUFMAN, Y.J. Atmospheric corretion algorithm for NOAAAVHRR products: theory and application. IEEE Transactions Geoscience Remote Sensing, Picataway, v.30, n.2, p.231-48, 1992.

VERZIGNASSI, J.R.; FERNANDES, C. D. Doenças em forrageiras: gado de corte divulga. Campo Grande: Embrapa Gado de Corte, 2001. v.50, 3 p. 